\title{
Development of a Water Cherenkov Detector Prototype with Wavelength Shifters and Silicon Photomultiplier Readout
}

\author{
Abaz Kryemadhi*, Brandon Weindorf, Aeowyn Kendall, Trieu Luu, Harry Hawbecker \\ Dept. of Math, Physics, and Statistics, Messiah College, Mechanicsburg, PA 17055, USA \\ E-mail: akryemadhi@messiah.edu
}

\begin{abstract}
Indirect high energy cosmic and gamma ray experiments require cost effective detectors and a large coverage area. Water Cherenkov detectors with large photomultiplier tubes have been commonly used for these experiments. While the photomultiplier tubes have good dynamic range and could be manufactured to larger sizes, their main drawbacks are high voltage to operate and sensitivity to magnetic fields. Silicon photomultipliers are the solid state counterparts which are insensitive to magnetic fields and operate at lower voltage, but have smaller area. At a time when solid state silicon photomultiplier vendors are growing and photomultiplier tubes declining, it is of paramount importance to develop alternative cost-effective readout schemes for future experiments. We developed a prototype water Cherenkov detector with wavelength shifting fibers and silicon photomultiplier readout. We report on the performance of this prototype for cosmic ray muons. We used Geant4 simulations to extrapolate performance at larger sizes
\end{abstract}

36th International Cosmic Ray Conference -ICRC2019-

July 24th - August 1st, 2019

Madison, WI, U.S.A.

\footnotetext{
*Speaker.
} 


\section{Introduction}

Indirect cosmic and gamma ray experiments are contingent on large light yield Cherenkov detectors for precision measurements of energy and arrival angle of showers (see for instance references $[1,2])$. Photomultiplier tubes have been and continue to be the main photon detection technology for these experiments because they can be manufactured in large sizes hence higher light yields. The drawbacks of these devices is their higher voltage of operation and a limited number of vendors producing them [3]. Silicon photomultipliers (SiPMs) are the solid-state equivalents to phototubes which operate at lower voltage. The main drawbacks of SiPMs is their small size and higher dark rate. In order to circumvent their small size we developed a water Cherenkov detector (WCD) where wavelength shifting fibers (WLS) are inserted in the water volume and the SiPMs detect the light at the end of the fibers. We characterize the detector's performance and report on possible usage for indirect experiments.

\section{Experimental Setup}

Our detector prototype is an open top cylindrical polyethylene tank with diameter of $71 \mathrm{~cm}$ and height of $106 \mathrm{~cm}$. The tank was filled with about 380 liters of water which underwent 1 micron and 5 micron filtering followed by reverse osmosis and de-ionization. All the inner surfaces of the tank were wrapped with Tyvek 1085D chosen for its highly reflective and diffuse surface. The tank was translucent so it was wrapped on the outside by layers of light proof plastic and black cloth as shown in figure 1(Left). We used BCF-92 multi-cladded blue to green WLS fibers from Saint-Gobain with a diameter $5 \mathrm{~mm}$ and length of $1 \mathrm{~m}$, chosen for their highest trapping efficiency. Four WLS fibers were bundled together and placed along the height at the center of the tank. The bottom part of each fiber was wrapped in teflon tape to reflect the captured photons back into the fiber. A silicon photomultiplier (SiPM) array from SensL (2x2 ArrayJ60035) detects the photons from fiber ends at the top. The readout board was custom made as described in ref [4]. The waveforms from the detector were captured by CAEN V1720 digitizer and data processed with CERN ROOT software. A sample Cherenkov pulse is shown in figure 1(Right).
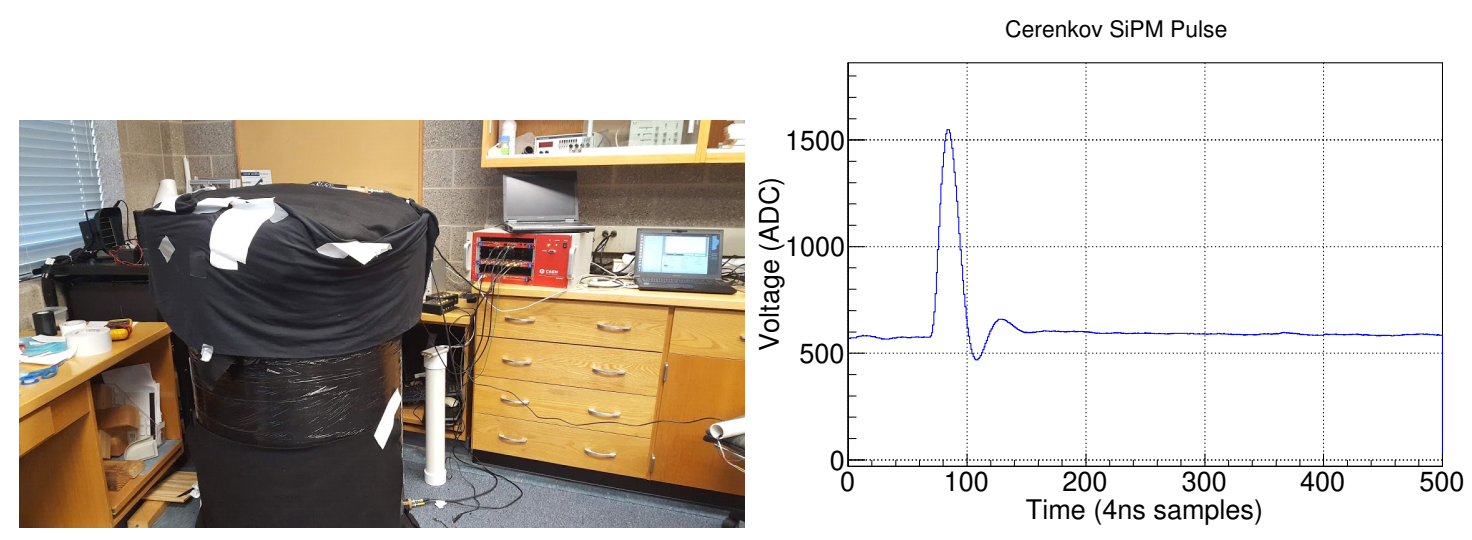

Figure 1: (Left) A picture of our setup. The 380 liter barrel is wrapped in black, the scintillator paddles are on top and bottom and the readout is using CAEN V1720 digitizer. (Right) A typical pulse from the detector. 


\section{Measurements \& Analysis}

The captured pulses were integrated to measure the charge and consequently the light yield. In a previous work we determined the single photon calibration of $\sim 50$ charge ADC ( $\sim 2$ voltage ADC). The dark rate charge spectrum is shown in figure 2(Left) where one observes that contributions beyond 1000 charge ADC ( 20 photoelectrons (p.e)) are negligible. Figure 2(Right) shows the charge spectrum obtained when a cosmic ray muon passes through our detector. Cosmic ray muons were triggered by plastic scintillators on top and bottom of the detector. The plastic scintillators had a length of $25 \mathrm{~cm}$, a width of $25 \mathrm{~cm}$, and a thickness of $1 \mathrm{~cm}$. The transition from dark rate to cosmic ray muons contributions is observed in the charge spectrum at around 1000 ADC. This demonstrates the capabilities of the detector to resolve signal from noise and establishes a threshold for a standalone operation which one would expect if it were part of an array. The wider range of the spectrum is primarely due to different paths travelled through the water volume and detector resolution.
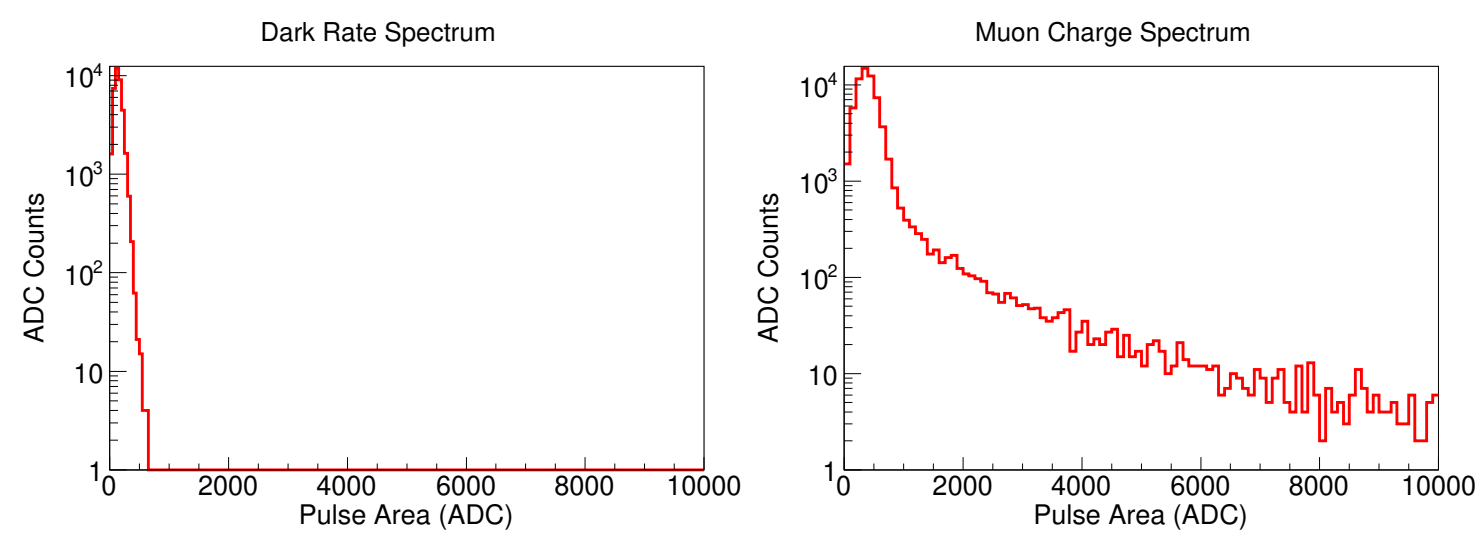

Figure 2: (Left) Dark spectrum integral ADC. (Right) Cosmic Ray muon triggered spectrum integral ADC.

We used Geant4 [5] to simulate our detector. The wide spectrum in figure 2(Right) makes it difficult to compare the absolute light yields between data and simulations. This however is not crucial since practically most experiments use calibration with respect to another data point, for instance a vertical muon charge. The focus of the simulations was instead on trends in light yield for different particle hit location, different fiber configurations, and different detector sizes.

Figure 3(Left) shows how the light yield varies in simulations as a function of radius of vertical cosmic ray muon hit for two different fiber placements. In one configuration the four fibers were all bundled together (configuration which we used for the data), in the other configuration the four fibers are separated to form a square where each fiber is located $\approx 25 \mathrm{~cm}$ from the center. The light yield does not change appreciably as we vary the hit location; this is due to diffuse nature of the Tyvek surface and the high absorption length of water. We observe a slightly higher light yield near fiber placement locations which is expected due to higher probability of direct light absorption by the fibers.

We repeated the simulations for a detector with $300 \mathrm{~cm}$ diameter and $300 \mathrm{~cm}$ height where four $300 \mathrm{~cm}$ long fibers are used and the result is shown in figure 3(Right). In the case of the separated 
fibers they were arranged in a square where each fiber is now a distance of $\sim 100 \mathrm{~cm}$ from the center.

There is no substantial advantages in the light yield between two different fiber placements and since the light yield does not change appreciably even for larger diameter detectors, the bundled fiber configuration at the center is preferred because of the simpler construction process.
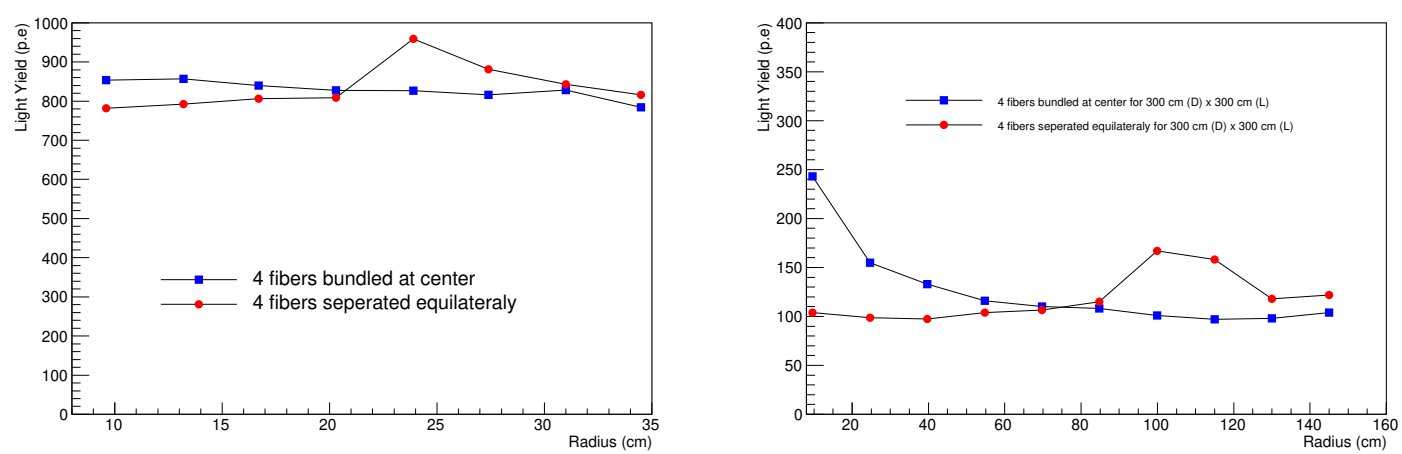

Figure 3: (Left) Light Yield vs impact radius for a vertical muon for our prototype size. (Right) Light Yield vs impact radius for a vertical muon for a detector size of $300 \mathrm{~cm}$ diameter and $300 \mathrm{~cm}$ height.

Additionally, we simulated the light yield for a detector the size of an Auger Collaboration's surface detectors with a diameter of $360 \mathrm{~cm}$ and a height of $120 \mathrm{~cm}$ where we changed the number of $5 \mathrm{~mm}$ fibers from 1 to 64 . The lengths of the fibers are the height of the detector. The range of fibers was chosen to match the standard off the shelf arrays from SiPM vendors. We observed that the light yield for a $4 \mathrm{GeV}$ vertical muon hitting the detector at $\sim 70 \mathrm{~cm}$ from the center increases approximately linearly with fiber number as:

$$
\operatorname{LightYield}(p . e)=30+3 N_{\text {fibers }}
$$

\section{Conclusion}

We constructed a WCD using WLS fibers and SiPM readout where we were able to separate the cosmic ray muon signals from the dark rate noise. The detector could be used as a standalone Cherenkov detector in an array of detectors by imposing a threshold trigger. Geant 4 simulations were used to see whether keeping the fibers bundled in one place or separating them brought any advantages. Since there was no significant changes between the two a bundled fiber configuration is preferred for simplicity of readout and construction. Simulations showed that light yield does not vary appreciably as a function of vertical muon hit even for larger size detectors due to diffuse reflectivity of Tyvek and large absorption length of water. Simulation showed that light yield increased linearly with number of fibers as: $\operatorname{LightYield}(p . e)=30+3 N_{\text {fibers }}$. While the light yield is smaller than large area PMTs within the configuarations we considered some further work will be explored to improve performance. These WCD detectors could be used at this phase as part of an outrigger array to complement the main array in order to increase sensitivity for high energy particles. 


\section{Acknowledgement}

We acknowledge NASA Pennsylvania Space Grant Consortium and Messiah College Scholar Chair Program.

\section{References}

[1] The Pierre Auger Collaboration, The Pierre Auger Cosmic Ray Observatory, Nuclear Instruments and Methods in Physics Research A, 798, 2015, 172-213

[2] T. DeYoung for the HAWC Collaboration, The HAWC observatory, Nuclear Instruments and Methods in Physics Research A, 692, 2012, 72-76

[3] R. G. Wagner,et al., The Next Generation of Photo-Detectors for Particle Astrophysics, in: As-tro2010: Technology Development Papers, volume2010 ofThe Astronomy and Astrophysics DecadalSurvey, pp. 59

[4] A. Kryemadhi, L. Barner, A. Grove, J. Mohler, A. Roth, A LYSO crystal array readout by silicon photomultipliers as compact detector for space applications, Nuclear Instruments and Methods in Physics Research A, 912, 2018, 93-96

[5] S. Agostinelli et al., Geant4 a simulation toolkit, Nuclear Instruments and Methods in Physics Research A, 506(3), 2003, 250-303 